\title{
Sever Rhabdomyolysis With Normal Renal Function Test in a 29-Year Old Who is Known to have RYR1 Mutation
}

\author{
${ }^{1}$ Roa A. Ali, ${ }^{2}$ Hassan Naeem \\ General Medicine Department \\ Kettering General Hospital \\ Northamptonshire, United Kingdom
}

\begin{abstract}
Ryanodine receptor gene (RYR-1) is a gene encode calcium release channel in skeletal muscle , mutation in this gene lead to rare type of inherited congenital myopathy which is associated with rhabdomyolysis and fatal malignant hyperthermia . We report a case of a 29 year old male known to have sporadic RYR-1 mutation who presented with myalgia and fatigue post upper respiratory tract infection symptoms during COVID19 pandemic .His Creatine Kinase was markedly elevated without evidence of acute kidney injury which is usually uncommon. We also discuss in this case importance of checking CK during COVID19 pandemic . There is no cure for RYR-1 mutation but proper genetic counselling and patient's education about precipitating factor for malignant hyperthermia will prevent serious complication which can lead to death.
\end{abstract}

Keywords:- Rhabdomyolysis with normal renal function , RyR1 mutation and Malignant Hyperthermia.

\section{$>$ Objective:}

Rare disease

\section{$>$ Background:}

Ryanodine receptor (RYR-1) mutation is a rare genetic congenital myopathy that leads to dysfunctional muscle contraction, weakness, susceptibility to malignant hyperthermia ,central core disease ( CDC ) and rhabdomyolysis. The RYR1-1 gene is located on the long arm of chromosome 19 .It regulates production of a protein that plays an essential role in cellular regulation of calcium homeostasis into ryanodine receptor calcium channel (RYR-1 in the sarcoplasmic reticulum of skeletal muscle . it also modulates multiple intracellular signalling pathways which affects muscle maturation and contraction.(1)

\section{INTRODUCTION}

A 29 year gentleman presented with myalgia and generalised tiredness. He have been found to have a Creatine Kinase (CK) of 30,000 u/l .

\section{CASE PRESENTATION}

This patient is known to have Ryanodine receptor ( RYR-1 ) mutation. He was diagnosed at age of 19 year old. $\mathrm{He}$ had been extensively investigated in the past for chronic back and progressive widespread myalgia including: Thigh muscle MRI, electromyography (EMG), muscle biopsy and genetic testing which confirmed mutation of the RYR-1 receptor.

He presented with a history of severe generalised fatigue and leg cramps for one week which affect patient's usual activity. There was no history of fever, recent exertion, excess alcohol, prolonged fasting, sedative or other medications. However, there was a significant history of flu like illness including cough, coryza and sore- throat 10 days ago hence he was initially isolated to rule out COVID-19 . There was no family history of RYR-1 myopathy.

On observations and physical examination ,he was looking unwell fully conscious, oriented, tachycardic with heart rate: 120 beat per minute, his blood pressure was 120/70. He has normal respiratory rate ,saturation and was afebrile .There was widespread muscle tenderness specially in lumbar region and calf muscles bilaterally.

Biochemistry investigations showed a raised total Creatine Kinase ( CK ) level of 30,000 U/L (normal level 40-320 U/L), normal CRP and ESR and negative blood culture. Urinalysis revealed haemoglobinuria and a few red blood cells (normal range 0-5). Myoglobin in the urine was not assessed. Serum electrolytes and renal function were consistently normal throughout admission. Due to history of flu symptoms he had two swab of SARS-COV-2 RNA which was negative .

\section{MANAGEMENT AND OUTCOME:}

Treatment consisted mainly of extensive hydration . He received a two-litre bolus of $0.9 \%$ saline, followed by one litre, six hourly. His urine output remained around 300 $\mathrm{ml} / \mathrm{hr}$. However, the CK decline was slow compared to symptoms resolution. 
The patient had previous admissions with rhabdomyolysis usually needed two days of intravenous fluids until the CK fell to than $1000 \mathrm{U} / \mathrm{l}$, but on this admission, despite aggressive rehydration, the CK dropped to $5,000 \mathrm{U} / \mathrm{L}$ after 10 days .

\section{DISCUSSION}

In this case, patient is known to have Ryanodine receptor (RyR1) mutation congenital Myopathy which is associated with risk of malignant hyperthermia. He had mild respiratory symptoms 10 days before admission during COVID-19 pandemic .The nasopharyngeal specimen was obtained 10 days after onset of symptoms . Therefore, a negative swab will not exclude recent COVID 19 infection .and there was no other obvious precipitating factor for Rhabdomyolysis rather than Viral infection likely COVID -19 .The mechanism of rhabdomyolysis in influenza infection there are three different theories : ( 1 ) direct viral invasion of skeletal muscles, ( 2 ) release of cytokines causing direct muscle damage; (3) viral toxin causing myonecrosis leading to rhabdomyolysis. (2)

There are three types of inheritance : autosomal dominant trait , autosomal recessive Less commonly and finally some sporadic cases have been reported in the absence of any apparent family history of RYR1. There are many cases underdiagnosed, making it difficult to determine the true frequency in the general population therefore checking $\mathrm{CK}$ in patients who presented with muscle aches would help in early identification of rhabdomyolysis (3)

Malignant hyperthermia (MH): a rare life-threatening condition that is characterized by hypercapnia, fever , hypotension, hypoxemia, muscle rigidity, metabolic acidosis and if not treated eventually death after exposure to anesthetic agents and/or succinylcholine. There is multiple triggers for Rhabdomyolysis in patient with RYR1 mutation includes :physical exercise, especially performed under extreme environmental temperatures, viral infection, alcohol ,medical and recreational drugs.

\section{CONCLUSION}

We describe the details of this patient's sporadic case of RyR1 mutation with Rhabdomyolysis following viral infection. This report also describes a case of a markedly elevated CK without evidence of acute kidney injury which is usually uncommon .

Although RYR1 is rare and there is no effective therapy, accurate diagnoses and treatment of associated life threatening Rhabdomyolysis and providing adequate counselling about precipitating factors and information about serious complication is crucial .

\section{Genetic Counselling}

Any patient diagnosed with RYR1 mutation all first degree relative require evaluation for malignant hyperthermia risk, patient's and family requires explanation about serious symptoms and complication if exposed to general anethesia which can lead to death of the patient if not treated and to prevent such serious complication muscle biopsy is required to confirm the diagnose.(4)

\section{Learning Point :}

This case highlights the need to consider checking total CK levels in hospitalised patients with confirmed or suspected COVID 19 infection especially in populations who are prone to rhabdomyolysis.

Ethics: Consent was obtained by all participants mentioned in case report

\section{REFERENCES}

[1]. S.Shepherd, F Ellis, J Halsall, P Hopkins, R Robinson RYR1 mutations in UK central core disease patients: more than just the $\mathrm{C}$-terminal transmembrane region of the RYR1 gene

[2]. A Abdisamad M Ibrahim, Sukesh Manthri, Paolo K Soriano, Vikrampal Bhatti,and Chaitanya K Mamillapalli . Rhabdomyolysis Associated with InfluenzaA virus Infection 2018

[3]. S.Shepherd, F Ellis, J Halsall, P Hopkins, R Robinson RYR1 mutations in UK central core disease patients: more than just the $\mathrm{C}$-terminal transmembrane region of the RYR1 gene

[4]. Ayuk A. Anderson, Ph.D.; Rosemary L. Brown, Ph.D.; Brenda Polster, B.S.; Neil Pollock, M.B., Ch.B.; Kathryn M. Stowell, Ph.D. Identification and Biochemical Characterization of a Novel Ryanodine Receptor Gene Mutation Associated with Malignant Hyperthermia. Anesthesiology 2 2008, Vol.108, 208215

[5]. Michael F. Goldberg, MD, MPH, Co-Founder and President, RYR-1 , National organisation of rare disease .

[6]. Chatzizisis YS, Misirli G, Hatzitolios AI, Giannoglou GD.Eur J Intern Med. 2008 Dec;19(8):568-74. doi: 10.1016/j.ejim.2007.06.037. Epub 2008 Apr 28.PMID: 1904672 